\section{Robotically assisted transcranial Doppler with artificial intelligence for assessment of cerebral vasospasm after subarachnoid hemorrhage}

Shooka Esmaeeli, MD'; Courtney M. Hrdlicka, MD²; Andres Brenes Bastos, $\mathrm{MD}^{1}$; Jeffrey Wang, $\mathrm{MDCM}^{2}$; Santiago Gomez-Paz, $\mathrm{MD}^{3}$; Khalid A. Hanafy, MD, PhD² ; Vasileios-Arsenios Lioutas, $\mathrm{MD}^{2}$; Christopher S. Ogilvy, MD; Ajith J. Thomas, MD³ ${ }^{3}$ Shahzad Shaefi, MD, MPH'; Corey R. Fehnel, MD, $\mathrm{MPH}^{2}$; Ala Nozari, MD, PhD ${ }^{1,4}$

\footnotetext{
${ }^{1}$ Department of Anesthesiology, Critical Care and Pain Medicine, Beth Israel Deaconess Medical Center, Harvard Medical School, Boston, MA, USA

${ }^{2}$ Department of Neurology, Beth Israel Deaconess Medical Center, Harvard Medical School, Boston, MA, USA

${ }^{3}$ Neurosurgical Service, Beth Israel Deaconess Medical center, Harvard Medical School, Boston, MA, USA

${ }^{4}$ Department of Anesthesiology, Boston Medical Center, Boston University School of Medicine, Boston, MA, USA
}

\section{ORIGINAL ARTICLE}

Received: January 29, 2020

Revised: April 7, 2020

Accepted: April 14, 2020

\author{
Corresponding Author: \\ Shooka Esmaeeli, MD \\ Department of Anesthesia, Critical Care \\ and Pain Medicine, Beth Israel \\ Deaconess Medical Center, One \\ Deaconess Road, Rosenberg 470, \\ Boston, MA 02215, USA \\ Tel: +1-217-722-9510 \\ Fax: +1-617-754-2735 \\ E-mail: sesmaeel@bidmc.harvard.edu
}

\begin{abstract}
Background: Transcranial Doppler (TCD) ultrasound is an essential tool for the detection of cerebral vasospasm after subarachnoid hemorrhage $(\mathrm{SAH})$ but is limited by the availability of skilled operators. We examined the clinical feasibility and concordance of a robotically assisted TCD system with artificial intelligence with routine handheld TCD after SAH.

Methods: We evaluated TCD velocities in the anterior cerebral artery (ACA) and middle cerebral artery (MCA) of two patients with high-grade SAH and angiographic evidence of vasospasm. A single channel TCD device with a handheld diagnostic probe as well as a robotically assisted TCD device was used, the relationship of the two tests was evaluated using the bootstrap method of resampling for the concordance correlation coefficient (CCC) paired with a Pearson's correlation analysis, followed by a Bland-Altman plot.

Results: Patient 1 developed angiographic and TCD evidence of vasospasm in the proximal right MCA, but except for periods of disorientation remained neurologically intact. Angiographic, TCD and clinical evidence of ACA spasm occurred 6 days after ictus in patient 2. Robotically measured mean flow velocities were comparable to manual TCDs in the MCAs $(\mathrm{CCC}=0.83 ; 95 \%$ confidence interval $[\mathrm{Cl}]$, 0.42 to $0.96 ; P=0.001$ ) but not in the $A C A s(C C C=0.26 ; 95 \% \mathrm{Cl},-0.01$ to $0.71 ; P=0.26$ ).

Conclusion: Robotically assisted TCD system with artificial intelligence provides an alternative to manual TCD for assessment of MCA velocities in patients with SAH, expanding the availability of TCD to settings in which specialized clinicians are not available. Further studies for validation of this technology are warranted.
\end{abstract}

Keywords: Vasospasm, intracranial; Subarachnoid hemorrhage; Middle cerebral artery; Ultrasonography, Doppler, transcranial; Anterior cerebral artery; Artificial intelligence 


\section{INTRODUCTION}

Delayed cerebral ischemia (DCI), defined as cerebral infarction or neurological deterioration caused by cerebral vasospasm, is a significant cause of mortality and poor neurological outcome after nontraumatic subarachnoid hemorrhage (SAH) [1,2]. Although a growing body of evidence suggests that early brain injury, spreading depolarization, microcirculatory dysfunction and impaired autoregulation may be necessary for the pathophysiology of neurological injury after SAH [3], the association with large-vessel cerebral vasospasm remains undisputed. Indeed, symptomatic vasospasm with neurological deterioration has been reported to occur with an incidence of up to $40 \%$ after SAH. Consequently, timely application of therapies aimed at mitigating vasospasm remains an essential cornerstone of SAH management $[4,5]$. Oral administration of the calcium channel blocker nimodipine is considered standard of care in the prevention of cerebral vasospasm [6]. Intravenous milrinone according to the Montreal Neurological Hospital protocol [7] may also have beneficial effects, although well-controlled trials to verify its long-term effects on the neurological outcome are needed.

Early diagnosis of vasospasm is critically dependent on frequent and high-quality neurological examinations, but the noninvasive assessment of the blood flow velocity in the basal cerebral arteries or angiographic studies are also important [8]. Changes in diameter are inversely proportional to the mean velocity of the blood within the vessel, which can be measured using transcranial Doppler (TCD) sonography. High TCD velocities are generally associated with DCI, although some patients may remain asymptomatic despite TCD or radiographic evidence of vasospasm [9]. For angiographic vasospasm, the predictive value of TCD flow velocities in the middle cerebral artery (MCA) is exceptionally high. Indeed, a mean flow velocity $>200 \mathrm{~cm} / \mathrm{sec}$ in the MCA has a positive predictive value of $87 \%$ for angiographic spasm, whereas velocities of $<120 \mathrm{~cm} / \mathrm{sec}$ have a negative predictive value of as high as $94 \%$ [10]. A velocity increase of more than $50 \mathrm{~cm} / \mathrm{sec}$ within 24 hours is also a strong predictor for the development of symptomatic vasospasm [11].

A significant limitation of the routine use of TCD is that it is time-consuming and highly operator dependent [8]. Examination of patients using the handheld technique requires that the examiner has detailed three-dimensional knowledge of cerebrovascular anatomy and its variations, as well as the ultrasound technology and various TCD indicators of vasospasm. Recently, a robotically assisted ultrasound system that integrates ultrasound, robotics, and machine learning (Lucid ${ }^{\text {Tx }}$ M1 Transcranial Doppler Ultrasound System ${ }^{\oplus}$ and NeuralBot ${ }^{\mathrm{Tw}}$ System, Neural Analytics, Los
Angeles, CA) was approved by the Food and Drug Administration, providing a tool that can potentially eliminate the interoperator variability of TCD findings. This system combines TCD with a boxy headset containing robotic wands that employ artificial intelligence (AI) in the form of a machine learning software platform with algorithms to automatically adjusts the ultrasound probes in order to detect and insonate intracranial vasculature, particularly the MCA M1 segments. It can also readjust the probe position after patient movement to continue to insonate a previously obtained location. The use of robotic TCD has been explored in the literature among stroke and traumatic brain injury patients. For instance, in 2018, researchers reported using this system for noninvasive neuromonitoring by obtaining continuous, bilateral TCD recordings in critically ill brain injury patients. Nevertheless, the use of robotic TCD has not been explored among SAH patients $[12,13]$.

In this study, we aimed to examine the feasibility and accuracy of robotically assisted TCD for measuring cerebral blood flow velocities in the anterior cerebral artery (ACA) and MCA in patients with high grade $\mathrm{SAH}$ and angiographic vasospasm.

\section{METHODS}

\section{Patients}

After approval from Institutional Review Board at Beth Israel Deaconess Medical Center (Protocol number: 2019P001001), we reviewed the clinical data, the TCD results, and imaging reports from two patients, with high-grade $\mathrm{SAH}$ and intraventricular blood, requiring placement of external ventricular drain (EVD) for hydrocephalus management. Written informed consent by the patients was waived by the board. Both patients received standard monitoring and therapies, including nimodipine prophylaxis, optimization of their hemodynamics and volume status, as well as fever control and correction of metabolic and electrolyte disturbances.

\section{Patient 1}

A 47-year-old male developed a thunderclap headache without any known triggering factors. He awoke the next morning with a severe headache and mental status changes and was brought to the hospital by his family. A computed tomography (CT) scan of his head showed a modified Fischer 3 SAH (Fig. 1A), CT angiogram revealed a $4 \mathrm{~mm}$ anterior communicating artery aneurysm. The patient was then transferred to our hospital and was found to follow commands inconsistently (Hunt and Hess Grade III). His level of arousal deteriorated, and he was intubated for airway protection. An EVD was placed in the setting of acute hydrocephalus. 
He was admitted to the intensive care unit, and an angiogram was performed the next day, with coiling of the aneurysm.

\section{Patient 2}

A 60-year-old previously healthy male presented after acute onset of severe headache followed by vomiting and unresponsiveness (Hunt and Hess Grade V). A head CT showed extensive and thick SAH with intraventricular hemorrhage, a modified Fischer 4 $\mathrm{SAH}$ (Fig. 1B) with effacement of the basal cisterns and foramen magnum concerning for uncal and tonsillar herniation. The patient was started on nicardipine infusion for blood pressure control, and a right frontal EVD was placed. CT angiogram showed an anterior communicating artery aneurysm (Fig. 2). Cerebral angiography confirmed the aneurysm, which was then embolized without complication. Eight days after ictus, TCD showed moderately increased mean flow velocities in the left MCA (mean 106 $\mathrm{cm} / \mathrm{sec}$ ), and although it did not reach the threshold for vasospasm, the interval change compared to the day prior raised concern for evolving vasospasm. Cerebral angiogram showed bilateral severe ACA (A2) vasospasm, which improved after selective arterial injection of nitroglycerin and verapamil.
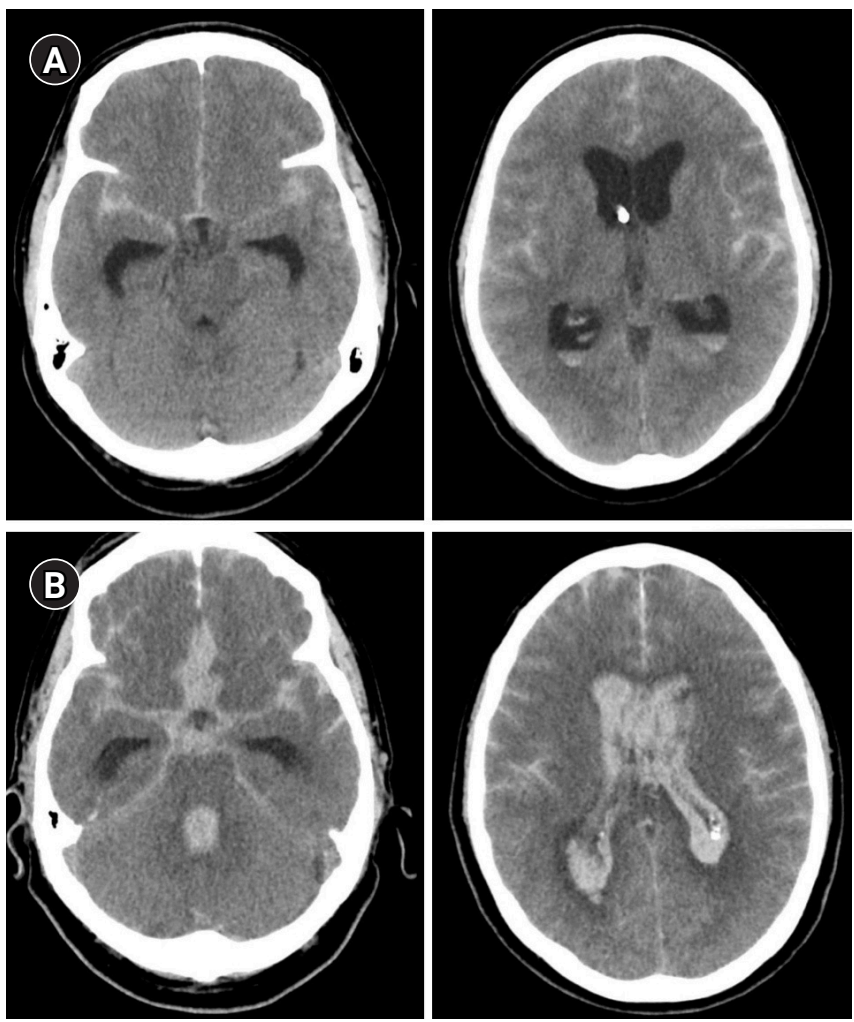

Fig. 1. Computer tomography imaging of $(A)$ patient 1 and (B) 2 showing diffuse thick subarachnoid hemorrhage with intraventricular blood.

\section{TCD imaging}

Manual TCD imaging was completed by vascular neurologists using a single channel TCD device with a handheld diagnostic probe (ST3 Transcranial Doppler, Spencer Technologies, Redmond, WA, USA). Once applied to the head, the Lucid ${ }^{\circledR}$ Robotic System (Neural Analytics) with AI and machine learning algorithm automatically searched for and detected the bilateral MCAs independent of an operator. The device also allowed for identification and measurement of TCD velocities in other cerebral vessels, but required control and adjustment of the TCD probes through a computer screen by a vascular technologist. The ACA measurements were, hence, not fully automated. We compared results of attempted insonation of the terminal internal carotid artery (ICA), MCA, ACA via the transtemporal window. We did not compare attempted insonation of the posterior cerebral artery, also visible via the transtemporal window. We recorded all suitable quality waveforms along the terminal ICA and MCA. The robotic system consistently automatical-
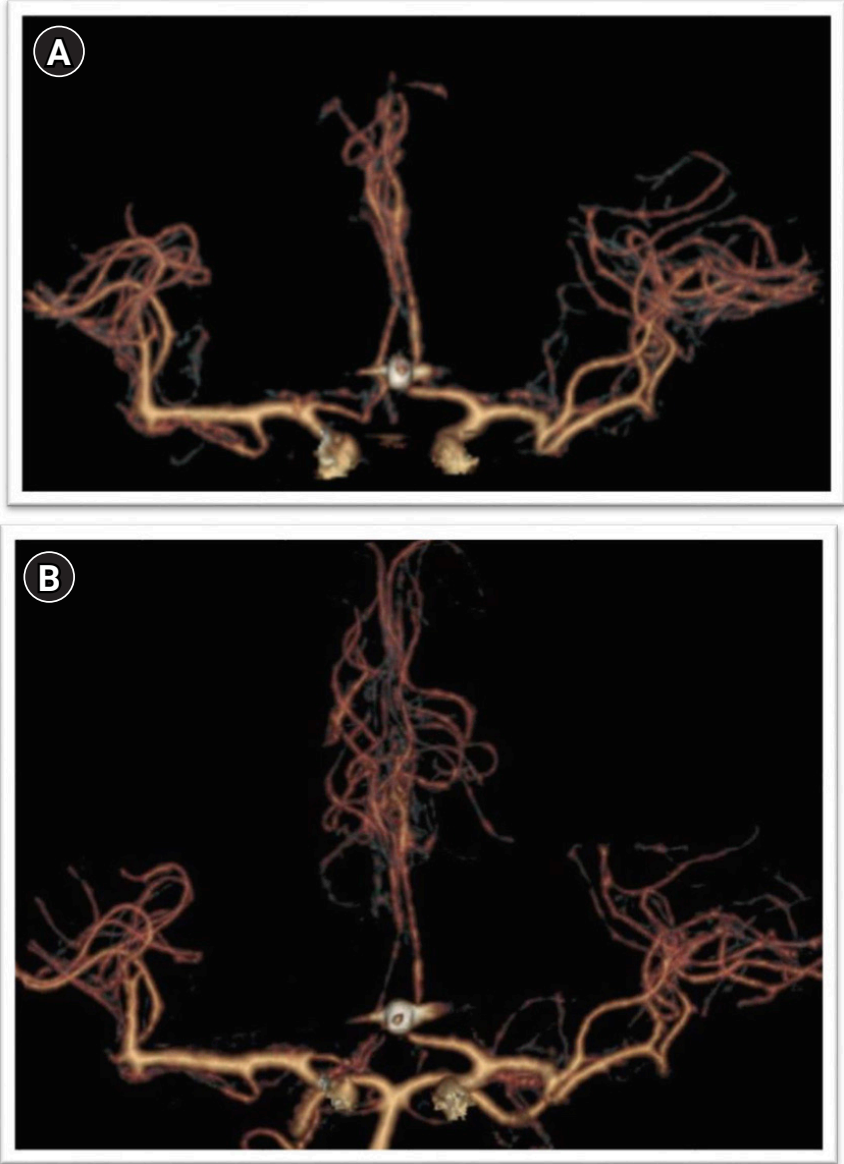

Fig. 2. Angiographic findings in patient 2, (A) initially demonstrating normal calibers in all cerebral vessels and (B) significant vasospasm in bilateral anterior cerebral arteries 6 days after ictus. 
ly located at least one (and often several) depths of the MCA. Manual manipulation of the robotic TCD probe was then performed to optimize signals, check additional depths, and to locate ACA signals.

\section{Statistical analysis}

The dependent concordance correlation coefficient (CCC) was calculated to measure the agreement between robotic and manual TCD methods. A resampling method was used for interference on dependent CCCs. The bootstrap method was used as the choice of resampling approach [14]. A set of 1,000 bootstrap samples was used as a proxy for independent samples and the bias-corrected and accelerated bootstrap confidence intervals (CIs) were reported. For quantitative comparison of the measured velocities, and to interpret the strength of agreement the CCC was paired with a Pearson's correlation analysis using the bootstrap approach [15]. Further, to visually assess the agreement between these methods, we pooled data from the MCA and ACA measurements separately, and plotted the differences between measurements at each time point against their means based on Bland and Altman [16] method of assessing agreement between methods of measurement with multiple observations per individuals. Data were analyzed using BlandAltmanLeh [17] and epiR [18] packages in R [19], and a $P$ value of less than 0.05 was considered significant.

\section{RESULTS}

\section{TCD findings}

For patient 1, manual and robotic TCD ultrasonographic studies were performed on the same day on three occasions, on postictus days 10,11 , and 12 (Fig. 3). The mean range of ICA/MCA depths insonated via the transtemporal window was $33 \mathrm{~mm}$ manually and $21 \mathrm{~mm}$ robotically per side. The highest mean flow velocities of each day's manual and robotic TCD were mostly concordant with few exceptions (Table 1).

For patient 2, manual and robotic TCD ultrasonographic studies were performed on the same day on two occasions, on postictus days 15 and 17 (Table 1). The mean range of ICA/MCA

(A)

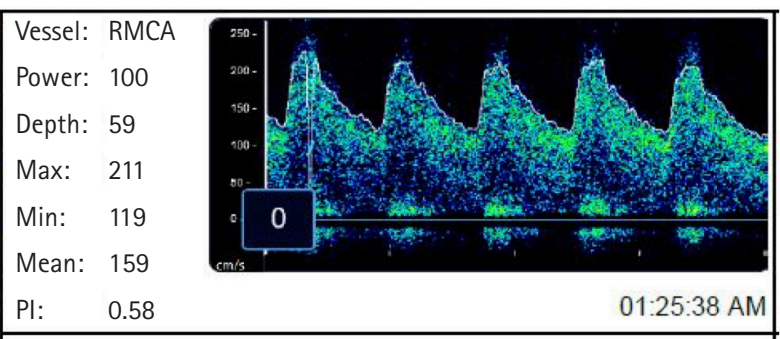

B

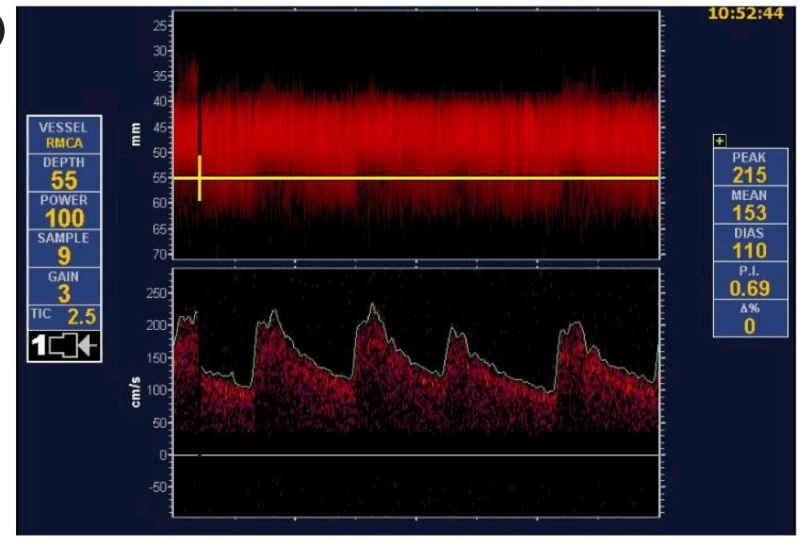

Fig. 3. (A) Robotic and (B) manual transcranial Doppler waveform of the right middle cerebral artery (MCA) of patient 1 on postictus day 10 demonstrating elevated right MCA mean flow velocity, consistent with vasospasm in both techniques.

Table 1. Mean flow velocities in anterior circulation reported by manual and robotic TCD imaging

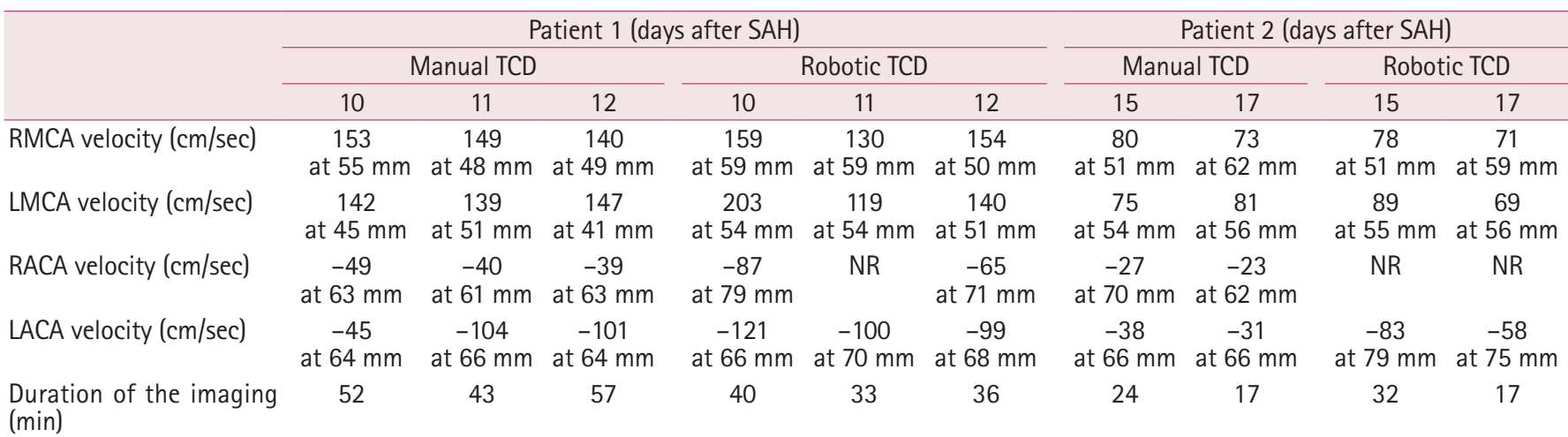

TCD, transcranial Doppler; SAH, subarachnoid hemorrhage; RMCA, right middle cerebral artery; LMCA, left middle cerebral artery; RACA, right anterior cerebral artery; NR, not reported; LACA, left anterior cerebral artery. 
depths insonated via the transtemporal window was $28 \mathrm{~mm}$ manually and $21 \mathrm{~mm}$ robotically per side. The average time required to conduct a complete TCD examination of the bilateral MCA and ACA vessels was 38.6 \pm 17 minutes with manual technique, and $30.8 \pm 8$ minutes for robotic technique (95\% CI, -13.6 to 29.2; $P=0.4$ ) (Table 1 ).

Representative plot of the robotic and manual TCD are presented in Fig. 4. Overall, robotically measured mean flow velocities were comparable to manual TCDs with AI assisted technology in the MCAs (CCC $=0.83$; $95 \% \mathrm{CI}, 0.42$ to $0.96 ; P=0.001$ ) but not in the ACAs $(\mathrm{CCC}=0.26$; $95 \% \mathrm{CI},-0.01$ to $0.71 ; P=0.26)$. The statistics for 1,000 bootstrapped correlations applied to the data is reported in Table 2 (Fig. 4). Bland-Altman plot showed overall agreement between measurements from both techniques, with most of the variables within $95 \% \mathrm{CI}$ indicating agreement between the two methods for both ACA and MCA velocities (Fig. 5).

\section{Patient outcome}

Patient 1 was extubated soon after the coiling of his aneurysm with his neurological examination returning rapidly to his baseline, apart from intermittent confusion and delirium. He remained neurologically intact and was discharged to his home in stable condition 17 days after his bleeding (modified Rankin Scale score $=0$ ). Patient 2 improved gradually after intraarterial vasospasm therapy and initiation of milrinone. He was successfully extubated 11 days after admission. Milrinone was weaned as his vasospasm improved, and his EVD was removed 1 week later (on hospital day 18). The patient was discharged to a rehabilitation facility in stable condition with moderate cognitive deficits and moderate left hemiparesis (modified Rankin Scale score $=4$ ).

\section{DISCUSSION}

We report that robotically assisted TCD with AI is a feasible alternative to the standard handheld technique for obtaining flow ve-

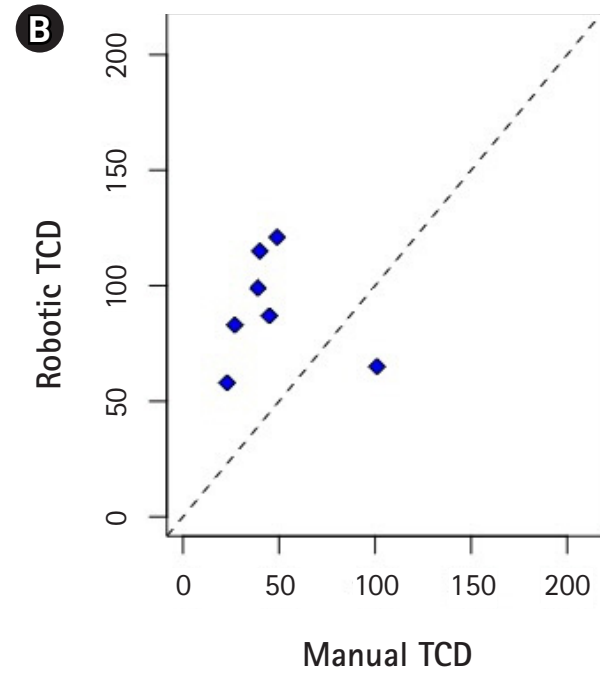

Fig. 4. (A) Moderate agreement between transcranial Doppler (TCD) findings using robotic technique and TCD findings using manual technique for the middle cerebral artery (concordance correlation coefficient=0.83; 95\% confidence interval [Cl], 0.42 to 0.96 ). (B) Poor agreement between TCD findings using robotic technique and TCD findings using manual technique for the anterior cerebral artery (concordance correlation coefficient $=0.26 ; 95 \% \mathrm{Cl},-0.01$ to 0.71 ).

Table 2. Comparison between the mean flow velocities in anterior circulation reported by manual and robotic transcranial Doppler imaging

\begin{tabular}{|c|c|c|c|c|}
\hline & $\begin{array}{l}\text { Average bootstrap estimate of } \\
\text { concordance coefficient }\end{array}$ & Bootstrap standard error & $\begin{array}{c}\text { Bootstrap 95\% BCa confidence } \\
\text { interval }\end{array}$ & Bootstrap estimates of bias \\
\hline$\overline{M C A}$ & 0.83 & 0.12 & 0.42 to 0.96 & -0.01 \\
\hline ACA & 0.26 & 0.20 & -0.01 to 0.71 & -0.001 \\
\hline
\end{tabular}

Bootstrapping was applied with $n=1,000$ to obtain concordance coefficient results.

$\mathrm{BCa}$, bias-corrected and accelerated; $\mathrm{MCA}$, middle cerebral artery; $\mathrm{ACA}$, anterior cerebral artery. 
(A)

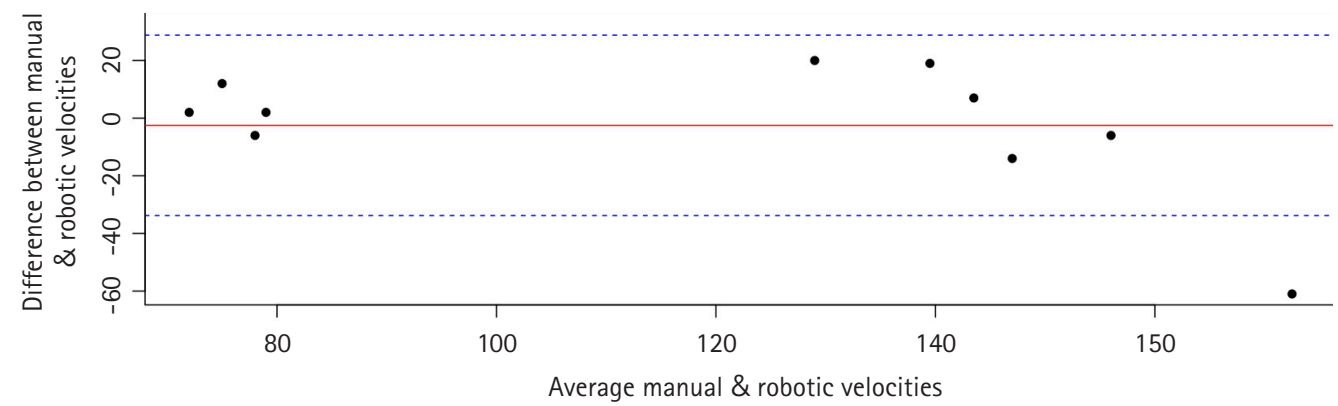

B

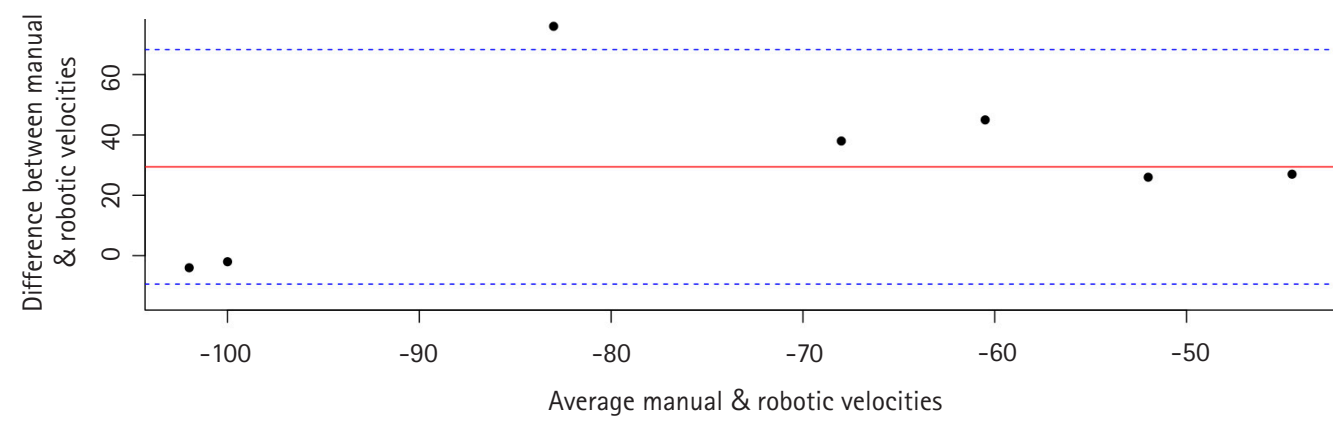

Fig. 5. Bland-Altman plot shows no proportional bias, indicating agreement between the two methods for the (A) middle cerebral artery and (B) anterior cerebral artery.

locities in patients with cerebral vasospasm after high grade $\mathrm{SAH}$. Despite significant vasospasm in both patients, MCA and ACA velocities could be identified without significant delay, and the AI guided MCA velocities were comparable to those obtained with the manual technique by expert clinicians.

The importance of early TCD monitoring in patients with highgrade $\mathrm{SAH}$ is demonstrated by the previous findings that elevated velocities may precede clinical symptoms by 24 to 48 hours $[20,21]$, which can signal the clinicians to intensify preventive strategies and implement therapeutic measures as soon as signs of clinical deterioration are noted. Clinicians should be alerted when a rapid increase in the TCD velocities is recorded, or when a high absolute mean flow velocity is obtained. The latter is indeed highly specific for angiographic vasospasm. As an example, in a study of 34 consecutive patients with SAH, Sloan and associates reported a specificity of $100 \%$ and sensitivity of $59 \%$ for the detection of angiographic spasm with a threshold of the mean flow velocity of $>120 \mathrm{~cm} / \mathrm{sec}$ [22].

The Lucid Robotic System combines TCD with a headset containing robotic wands and uses machine learning to find the best cranial window and insonation angle based on patterns in the data it gathers (Fig. 6). In contrast to traditional ultrasound systems, the robotic system does not require the presence of a trained professional to locate MCA waveforms. Use of robotic TCD may in-

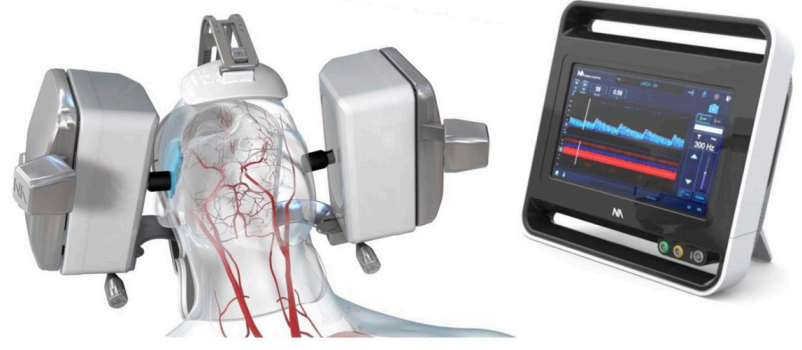

Fig. 6. The robotically assisted transcranial Doppler system used in this study (Neural Analytics Lucid Robotic System. Credit: Neural Analytics).

crease the availability of this technique, and therefore the ability to implement early interventions to mitigate the potential neurological consequences of vasospasm and cerebral ischemia. In its current version, the $\mathrm{AI}$ allows automatic detection of flow velocities only in the bilateral MCAs. This is, nevertheless, clinically important because of the high positive and negative predictive values for angiographic spasm for mean MCA flow velocities [10], whereas the predictive value of TCD velocities in other vascular territories is not as strong [23]. The robotic system does, nevertheless, allow a full examination of other intracranial vessels when directed and 
controlled by an examiner, although the current version of its self-learning software is limited to only the bilateral MCAs. It is important to note; however, that our data failed to show a significant correlation or concordance between the robotic and manual TCD findings in the ACAs, and this lack of correlation may also exist for other intracerebral vessels not examined in the current report. Nonetheless, given the absence of proportional bias in the Bland-Altman plot, an agreement between the methods appears to exist even for the ACA velocities. Further studies with larger sample size are therefore warranted to decipher these findings.

It is important to note that although the two methods were performed on the same day, the discrepancies in the mean flow velocities in ACAs were higher than MCAs. In addition to underlying physiologic changes, the discrepancy in ACA flow velocities can indicate an inherent difference in training and technical skills between the two operators: a vascular neurologist versus a vascular technologist. It can also imply technical limitations with the manually controlled ACA measurements with the robotic TCD: the AI machine learning technology was only available and used for the MCAs, whereas the ACA measurements remained operator dependent.

Limitations of the TCD imaging in SAH patients include an inability to insonate intracranial vessels in $10 \%$ to $20 \%$ of patients. Indeed, Seidel et al. [24] reported insufficient acoustic temporal bone window in about $20 \%$ of the studied population. A significant limitation that is inherent to the TCD technique is related to the measurement of a cerebral blood flow velocity as a surrogate for cerebral blood flow. Cerebral blood flow velocity is, however, proportional to the cerebral blood flow only if the cross-sectional vessel area remains constant [25]. Moreover, the spatial resolution of TCD is limited for the posterior circulation [26], which, combined with its relatively low sensitivity, makes clinical examination the most crucial factor in decision making for patients with posterior circulation vasospasm.

The robotically assisted TCD system used in this study has additional limitations such as inability to insonate posterior circulation with its current headset, as well as its large size and weight (Fig. 3), which may not be well tolerated by some patients, particularly after craniotomy or cervical spine injury. Moreover, healthcare providers need to be trained to initiate the robot and apply the head device to the patient. Importantly, this technology is currently only capable to find and assess the MCA velocities, as it is supported with our findings. To assess other cerebral vessels the technology needs upgrading. Despite these limitations, the robotically assisted TCD system can be useful in institutions without or with limited access to professionals trained to obtain manual TCD waveforms. Furthermore, this technology allows for extended monitoring of a single vessel, which could allow for visualization of immediate effects of any treatment that is employed to enhance cerebral blood flow and manage vasospasm after SAH. Needless to say, even with robotic TCD a skilled clinician is always required for evaluation and interpretation of the data.

Although our results are compelling, it is important to discuss the limitations of our study. First, we have a very small sample size in this study which may affect our results and findings. Second, in this study the robotic and manual TCD measurements were often conducted many hours apart, albeit on the same day. Although unlikely, any physiological changes over the course of the day may have affected the velocities and lead to the discrepancy between data. These and other limitations described above will need to be addressed in future studies.

In our two patients, robotically assisted TCD with AI was feasible for evaluation of MCA waveforms in SAH-associated vasospasm and provided results that were overall comparable to manual TCD performed the same day. Further studies to assess the validity of this technology in SAH patients are warranted.

\section{ARTICLE INFORMATION}

\section{Conflict of interest}

No potential conflict of interest relevant to this article.

\section{ORCID}

Shooka Esmaeeli, https://orcid.org/0000-0001-7614-7074 Courtney M. Hrdlicka, https://orcid.org/0000-0001-5075-3869 Andres Brenes Bastos, https://orcid.org/0000-0002-0576-2659 Jeffrey Wang, https://orcid.org/0000-0003-4119-6524 Santiago Gomez-Paz, https://orcid.org/0000-0003-2283-3612 Khalid A. Hanafy, https://orcid.org/0000-0002-5979-1367 Vasileios-Arsenios Lioutas, https://orcid.org/0000-0002-8521-0036 Christopher S. Ogilvy, https://orcid.org/0000-0003-4600-8545

Ajith J. Thomas, https://orcid.org/0000-0003-4412-3152 Shahzad Shaefi, https://orcid.org/0000-0002-6832-3282 Corey R. Fehnel, https://orcid.org/0000-0003-1726-5809 Ala Nozari, https://orcid.org/0000-0002-5755-6347

\section{Author contributions}

Conceptualization: VAL, CSO, and AN. Data curation: SE, CMH, ABB, JW, and SGP. Formal analysis: SE, CRF, and AN. Visualization \& Writing-original draft: SE and AN. Writing-review editing: $\mathrm{CMH}, \mathrm{JW}, \mathrm{KAH}, \mathrm{VAL}, \mathrm{CSO}, \mathrm{AJT}, \mathrm{SS}$, and CRF.

\section{REFERENCES}

1. Francoeur CL, Mayer SA. Management of delayed cerebral 
ischemia after subarachnoid hemorrhage. Crit Care 2016;20: 277.

2. Kreiter KT, Copeland D, Bernardini GL, Bates JE, Peery S, Claassen J, et al. Predictors of cognitive dysfunction after subarachnoid hemorrhage. Stroke 2002;33:200-8.

3. Rowland MJ, Hadjipavlou G, Kelly M, Westbrook J, Pattinson KT. Delayed cerebral ischaemia after subarachnoid haemorrhage: looking beyond vasospasm. Br J Anaesth 2012;109:31529.

4. Schmid-Elsaesser R, Kunz M, Zausinger S, Prueckner S, Briegel J, Steiger HJ. Intravenous magnesium versus nimodipine in the treatment of patients with aneurysmal subarachnoid hemorrhage: a randomized study. Neurosurgery 2006;58:1054-65.

5. Daou BJ, Koduri S, Thompson BG, Chaudhary N, Pandey AS. Clinical and experimental aspects of aneurysmal subarachnoid hemorrhage. CNS Neurosci Ther 2019;25:1096-112.

6. Connolly ES Jr, Rabinstein AA, Carhuapoma JR, Derdeyn CP, Dion J, Higashida RT, et al. Guidelines for the management of aneurysmal subarachnoid hemorrhage: a guideline for healthcare professionals from the American Heart Association/American Stroke Association. Stroke 2012;43:1711-37.

7. Lannes M, Teitelbaum J, del Pilar Cortés M, Cardoso M, Angle M. Milrinone and homeostasis to treat cerebral vasospasm associated with subarachnoid hemorrhage: the Montreal Neurological Hospital protocol. Neurocrit Care 2012;16:354-62.

8. Sharma S, Lubrica RJ, Song M, Vandse R, Boling W, Pillai P. The role of transcranial Doppler in cerebral vasospasm: a literature review. Acta Neurochir Suppl 2020;127:201-5.

9. Schmidt JM, Wartenberg KE, Fernandez A, Claassen J, Rincon F, Ostapkovich ND, et al. Frequency and clinical impact of asymptomatic cerebral infarction due to vasospasm after subarachnoid hemorrhage. J Neurosurg 2008;109:1052-9.

10. Vora YY, Suarez-Almazor M, Steinke DE, Martin ML, Findlay JM. Role of transcranial Doppler monitoring in the diagnosis of cerebral vasospasm after subarachnoid hemorrhage. Neurosurgery 1999;44:1237-48.

11. Grosset DG, Straiton J, du Trevou M, Bullock R. Prediction of symptomatic vasospasm after subarachnoid hemorrhage by rapidly increasing transcranial Doppler velocity and cerebral blood flow changes. Stroke 1992;23:674-9.

12. O’Brien M, Ranjbaran M, Ilyas P, Scheidt M, Thorpe S, Nicolas $\mathrm{C}$, et al. Fully automated transcranial doppler ultrasound insonation of the MCA using a five degree of freedom robotically actuated probe system. Eur J Neurol 2018;25:56-57.

13. Zeiler FA, Smielewski P. Application of robotic transcranial Doppler for extended duration recording in moderate/severe traumatic brain injury: first experiences. Crit Ultrasound J
2018;10:16

14. Williamson JM, Crawford SB, Lin HM. Resampling dependent concordance correlation coefficients. J Biopharm Stat 2007; 17:685-96.

15. Altman DG. Practical statistics for medical research. 1st ed. Boca Raton: CRC press; 1990.

16. Bland JM, Altman DG. Agreement between methods of measurement with multiple observations per individual.J Biopharm Stat 2007; 17:571-82.

17. Lehnert B. BlandAltmanLeh: Plots (Slightly Extended) Bland-Altman Plots. R package version 0312015. In: Cran R-project [Internet]. Vienna (AU): R Foundation; 2015 [cited 2020 May 23]. Available from: https://CRAN.R-project.org/package $=$ BlandAltmanLeh .

18. Stevenson M, Heuer C, Marshall J, Sanchez J, Reiczigel J, Robison-Cox J, et al. epiR: Tools for the Analysis of Epidemiological Data. R package version 10-102019. In: Cran R-project [Internet]. Vienna (AU): R Foundation; 2019 [cited 2020 May 23]. Available from: https://CRAN.R-project.org/package = epiR

19. R Core Team. R: A Language and Environment for Statistical Computing. In: R Foundation for Statistical Computing [Internet]. Vienna (AU): R Foundation; 2018 [cited 2020 May 23]. Available from: https://www.R-project.org.

20. Sekhar LN, Wechsler LR, Yonas H, Luyckx K, Obrist W. Value of transcranial Doppler examination in the diagnosis of cerebral vasospasm after subarachnoid hemorrhage. Neurosurgery 1988;22:813-21.

21. Mizuno M, Nakajima S, Sampei T, Nishimura H, Hadeishi H, Suzuki A, et al. Serial transcranial Doppler flow velocity and cerebral blood flow measurements for evaluation of cerebral vasospasm after subarachnoid hemorrhage. Neurol Med Chir (Tokyo) 1994;34:164-71.

22. Sloan MA, Haley EC Jr, Kassell NF, Henry ML, Stewart SR, Beskin RR, et al. Sensitivity and specificity of transcranial Doppler ultrasonography in the diagnosis of vasospasm following subarachnoid hemorrhage. Neurology 1989;39:1514-8.

23. Suarez JI, Qureshi AI, Yahia AB, Parekh PD, Tamargo RJ, Williams MA, et al. Symptomatic vasospasm diagnosis after subarachnoid hemorrhage: evaluation of transcranial Doppler ultrasound and cerebral angiography as related to compromised vascular distribution. Crit Care Med 2002;30:1348-55.

24. Seidel G, Kaps M, Gerriets T. Potential and limitations of transcranial color-coded sonography in stroke patients. Stroke 1995;26:2061-6.

25. ClarkJM, Skolnick BE, Gelfand R, Farber RE, Stierheim M, Stevens WC, et al. Relationship of 133 Xe cerebral blood flow to middle cerebral arterial flow velocity in men at rest. J Cereb 
Blood Flow Metab 1996;16:1255-62.

26. Aries MJ, Elting JW, De Keyser J, Kremer BP, Vroomen PC. Cerebral autoregulation in stroke: a review of transcranial Doppler studies. Stroke 2010;41:2697-704. 